\title{
Clinical examination in back pain
}

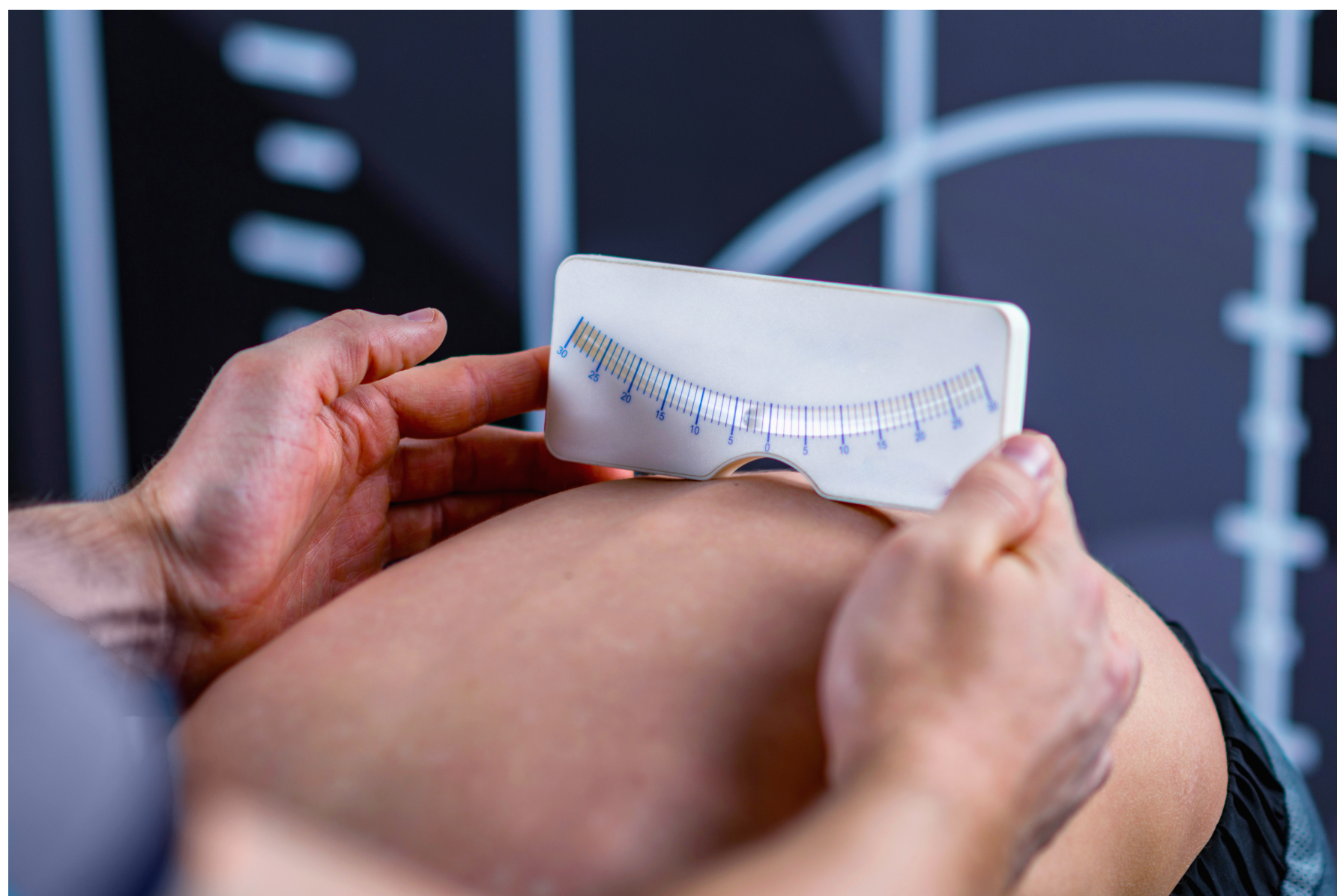

Die klinische Untersuchung des Rückenschmerzes

\section{Vavken J, Mameghani A}

Wirbelsäulenchirurgie, Kantonsspital Baden, Schweiz

\begin{abstract}
Back pain has become one of the most frequent sports-related health problems. Up to $80 \%$ of the Swiss population experience at least one episode per year up to several times per week. It affects athletes of all age groups and all levels of activity equally. The causes of acute and chronic back pain are plentiful, but can be easily appreciated with a thorough and comprehensive history, concise clinical examination, and adequate imaging.
\end{abstract}




\section{Zusammenfassung}

Auch im Sport zählen Lumbalgien mit zu den häufigsten gesundheitlichen Problemen. Zirka 80\% der Schweizer Bevölkerung leiden mindestens einmal pro Jahr bis mehrmals in der Woche an Rückenschmerzen. Betroffen sind Menschen aller Altersgruppen und Aktivitätslevel, die Ursachen sind vielfältig, anhand einer sorgfältigen Anamneseerhebung, klinischen Untersuchung und der passenden Bildgebung jedoch meist gut zu identifizieren.

\section{Einleitung}

Viele Leistungs- aber auch Hobbysportler klagen im Verlauf ihrer sportlichen Laufbahn über Rückenbeschwerden. Oft handelt es sich hierbei um Überlastungsschäden, die sich dann später als Degenerationen manifestieren können. Einerseits gibt es Sportarten, die vermehrt mit der Entstehung von Rückenschmerzen assoziiert sind, wie z.B. Gewichtheben, Bodybuilding und Rudern [1,2], andererseits scheint das Aktivitätslevel eine grosse Rolle zu spielen. Eine erhöhte Inzidenz von Rückenschmerzen zeigt sich sowohl bei extremen Bewegungsmuffeln als auch bei überdurchschnittlich sportlich aktiven Menschen [3,4].

Wichtig in der Behandlung des Rückenschmerzes ist, dass Symptome und Leidensdruck teils nur wenig mit der Bildgebung korrelieren, so dass die Klinik sowie der Anspruch des Patienten an seine Gesundheit/sein Aktivitätslevel in Diagnostik und Therapie führend sein sollten [5].

\section{Anamnese}

Das Gespräch mit dem Patienten und die Erhebung der Anamnese nehmen teils sehr viel Zeit in Anspruch, geben jedoch oft schon deutliche Hinweise auf das Erkrankungsbild und lenken die Untersuchungen in die richtige Richtung. Wichtig hierbei sind stets die Dauer der Symptome, Bewegungseinschränkungen, Paresen, Schmerzauslöser, Ausstrahlung, Vorerkrankungen sowie Erkrankungen der grossen benachbarten Gelenke zu erfragen. Auch die Blasen-/Mastdarmfunktion und Sexualfunktion müssen angesprochen werden, sowie andere «red flags» ausgeschlossen werden. (Tabelle 1)

Hilfreich sind auch Angaben des Aktivitätslevels bzw. ausgeübter Sportarten, da verschiedene Sportarten durchaus mit der Entstehung einzelner Pathologien vergesellschaftet sind. Die Spondylolyse z.B. ist durch die repetitiven hyperextendierenden und rotatorischen Krafteinflüsse bei Gewichthebern, Speerwerfern und Gymnasten überdurchschnittlich häufig anzutreffen [6,7]. Auch der Morbus Scheuermann ist mit der körperlichen Betätigung positiv korreliert. So ist aus Studien bekannt, dass der Kyphosewinkel mit dem Grad der Aktivität korreliert, sowie von der Art des Sportes (Gewichtheben, Gymnastik, Eiskunstlauf, Trampolin, Schwimmen, Fechten, Schiessen, Rudern, Ringen, Velofahren) abhängig sein kann [8,9].

\section{Gangprüfung}

Im Folgenden sollten die verschiedenen Gang- und Standarten geprüft werden. Der normale Gang kann dem Untersucher bereits viel Aufschluss über die Pathologie geben. Geachtet werden sollte auf Zügigkeit, Flüssigkeit, Schonhinken oder evtl. besondere Gangbilder wie einen Steppergang bei Fussheberparese sowie Trendelenburg- oder Duchenne-Hinken bei Hüftpathologien oder auch L5- 
Nervenwurzelkompressionssyndromen. Ein Funktionsverlust bei Hackenstand oder -gang oder eine isolierte Grosszehenheberschwäche können auf eine L4- oder L5-Symptomatik hinweisend sein, wobei hier differentialdiagnostisch auch immer an periphere Schädigungen des N. peroneus gedacht werden muss. Etwaige Probleme beim Zehenspitzenstand oder -gang können auf eine Kompression der S1-Wurzel zurückzuführen sein. Der Einbeinstand vermittelt schnell einen groben Überblick über die Hüftabduktion (Mm. glutei), wobei auch gerade bei Sportlern immer der Status nach operativem Hüftgelenkseingriff (Schädigung der Abduktoren durch z.B. lateralen Zugangsweg) erfragt werden sollte.

\section{Inspektion}

Im Anschluss daran erfolgt die Inspektion des Rückens. Hier ist die Dreidimensionalität der Wirbelsäule zu beachten. In der Sagittalebene wird die Doppel-S-Form in ihrer Ausprägung mit Lordosen (HWS und LWS) sowie Kyphosen (BWS uns Sacrum) betrachtet. Der normale LWS-Lordosewinkel beträgt 40 bis $60^{\circ}$ [9]. Eine Hyperlordose hier kann z.B. richtungsweisend für eine Spondylolisthese sein oder ein sog. flat back kann vorliegen nach vorangegangenen Wirbelsäuleneingriffen, aufgrund von Degenerationen oder kompensatorisch bei Hüftbeugekontrakturen.

Bei einer Hyperkyphose im BWS-Bereich sollte evtl. an einen Morbus Scheuermann gedacht werden. Die HWS zeigt im Normalfall eine Lordose zwischen 30 und $45^{\circ}$, gemessen von Deckplatte C3 auf Grundplatte C7. Streckstellungen sind meist aufgrund einer Schonhaltung anzutreffen, kyphotische Stellung können hinweisend auf massive Degenerationen oder Segmentinstabilitäten sein und sollten weiter mittels Röntgen und MRI abgeklärt werden, zumal Kyphosewinkel von über $10^{\circ}$ eng mit cervicaler Myelopathie assoziiert sind [10].

Von dorsal sollte der Winkel über die gesamte Wirbelsäule $0^{\circ}$ betragen (C7 plumb line auf die Mitte des Os sacrum). Geachtet wird hier vor allem auf Hinweise einer Skoliose mit evtl. vorhandenem Rippenbuckel oder Lendenwulst, auf Symmetrie der Taillendreiecke, Becken- oder Schulterschiefstand. Bei Verdacht auf eine Skoliose müssen - gerade im Wachstumsalter - weitere diagnostische Schritte wie Wirbelsäulenganzaufnahmen sowie Bendingaufnahmen eingeleitet werden, um die Komplexität dieses Krankheitsbildes erfassen und adäquat behandeln zu können. Hierzu sollte man den Patienten grosszügig an Kompetenzzentren weiterleiten.

Naevi und typische Behaarungen können auf dysraphische Störungen hinweisen und müssen dokumentiert werden.

\begin{tabular}{|c|c|c|c|}
\hline Conus-Cauda-Syndrom & Fraktur & vertebrale Infektion & Tumor \\
\hline Paresen & adäquates Trauma & Lumbalgie & bek. Tumorerkrankung \\
\hline Blasen-Mastdarmstörung & $\begin{array}{l}\text { Bagatelltrauma bei Osteopo- } \\
\text { rose, syst. Steroide }\end{array}$ & erhöhte Entzündungszeichen & B-Symptomatik \\
\hline Reithosenanästhesie & Antikonvulsiva & $\begin{array}{l}\text { Diabetes mellitus } \\
\text { St. } n \text {. Infiltration } \\
\text { Immunsuppression } \\
\text { i.v. Drogenabusus }\end{array}$ & $\begin{array}{l}\text { höheres Alter } \\
\text { nächtlicher Schmerz }\end{array}$ \\
\hline
\end{tabular}




\section{Palpation}

Im Rahmen der Inspektion können die Wirbelsäule sowie die paraspinale Muskulatur gut grob palpiert werden. Hier sollten vorerst in kraniokaudaler Richtung die Procc. spinosi abgetastet werden. Lokale Druckdolenzen auf den Procc. spinosi können richtungsweisend für eine Fraktur, einen Tumor, eine Spondylodiszitis oder auch eine Hernie sein, Druckdolenzen intraspinös nach einem Trauma könnten ein Indikator für eine komplexe/instabile Fraktur sein. Grossflächige Druckdolenzen hingegen werden unter anderem bei der Osteoporose angetroffen. Eine grob orientierende Perkussion mit der Faust entlang der Wirbelsäule kann weiteren Aufschluss über die Höhenpathologie geben, sollte jedoch stets vorsichtig durchgeführt werden. Stufenbildungen werden bei Spondylolisthesen getastet. Anschliessend können paramedial beidseits die Facettengelenke sowie die SI-Gelenke bds. geprüft sowie die paraspinale Muskulatur palpiert werden. Oft zeigen sich - am häufigsten im Bereich der HWS - schmerzhafte Myogelosen oder Triggerpunkte.

\section{Beweglichkeit}

Die Beweglichkeit im Bereich der HWS ist mit Abstand am grössten, gefolgt von der der LWS. Die BWS ist aufgrund der Verbindung zum Sternum über die Costae am starrsten, diese verminderte Beweglichkeit ist unter anderem auch der Grund für die geringere Inzidenz von thorakalen Diskushernien $(<1 \%$ aller Diskushernien) [11].

Pathologien im Bereich der LWS können mit einer reflektorisch verminderten Beweglichkeit derselben einhergehen. Eine schmerzhafte Reklination könnte hinweisend auf eine Spondylarthrose sein, oft zeigen sich dann auch Druckdolenzen über den Facettengelenken. Schnell zu testen, wenn auch sehr unspezifisch (DD Verkürzung der ischiocruralen Muskulatur, Hüftsteife, Armlänge, generelle Unbeweglichkeit) ist weiterhin der Finger-Boden-Abstand (FBA), der von Patient zu Patient sehr individuell ist, beim Gesunden jedoch unter $10 \mathrm{~cm}$ betragen sollte. Beim Vornüberbeugen mit gestreckten Beinen wird hier der Abstand der Fingerspitze des Mittelfingers zum Boden in cm gemessen. Währenddessen sollte auf die Symmetrie oder auch die Ausbildung eines Lendenwulsts (Verdacht auf Lumbalskoliose) oder Rippenbuckels (Verdacht auf Thorakalskoliose) geachtet werden. Das Schober-Mass und das Ott-Mass (Abbildung 1) können reproduzierbare Aussagen über die Mobilität machen. Weiterhin kann die Beweglichkeit der Wirbelsäule anhand der Neutral-Null-Methode gemessen werden. Wichtig hier sind in erster Linie weniger die absolut erreichten Werte als vielmehr etwaige Seitenunterschiede. (Tabelle 2) Die Beweglichkeit der HWS ist - wie oben bereits erwähnt - am höchsten. Hier gehört der Kinn-SternumAbstand zur Basisuntersuchung (Abstand Kinn - Sternum bei Inklination und bei Reklination). Eine schmerzhaft Reklination zeigt sich häufig bei Patienten mit Spondylarthrose, da hierdurch die Facettengelenke weiter komprimiert werden, was die Schmerzen akzentuiert. Weiterhin werden die Rotation und Seitneigung gemessen. Die Rotation zeigt sich bei Pathologien im Bereich der HWS oftmals schnell, meist einseitig eingeschränkt. Dies kann einerseits durch hartnäckige Myogelosen, schmerzhaft Schonhaltungen aber auch typischerweise bei einer C1/2-Arthrose der Fall sein. Ein positives SpurlingZeichen kann bei Cervicobrachialgie durch z.B. eine Diskushernie zur Höhenlokalisation beitragen. Hierzu wird beim sitzenden Patienten axialer Druck auf den Kopf bei HWS-Hyperextension und -lateralflexion zur betroffenen Seite hin ausgeübt. Dadurch verengt sich das Neuroforamen zusätzlich, bei bestehender Neurokompression entsteht/nimmt eine Brachialgie zu. 


\begin{tabular}{|l|l|l|l|}
\hline & Inklination/Reklination & Rotation & Lateralflexion \\
\hline HWS & $45 / 0 / 45$ & $80 / 0 / 80$ & $45 / 0 / 45$ \\
\hline BWS/LWS & $120 / 0 / 30$ & $45 / 0 / 45$ & $40 / 0 / 40$ \\
\hline
\end{tabular}

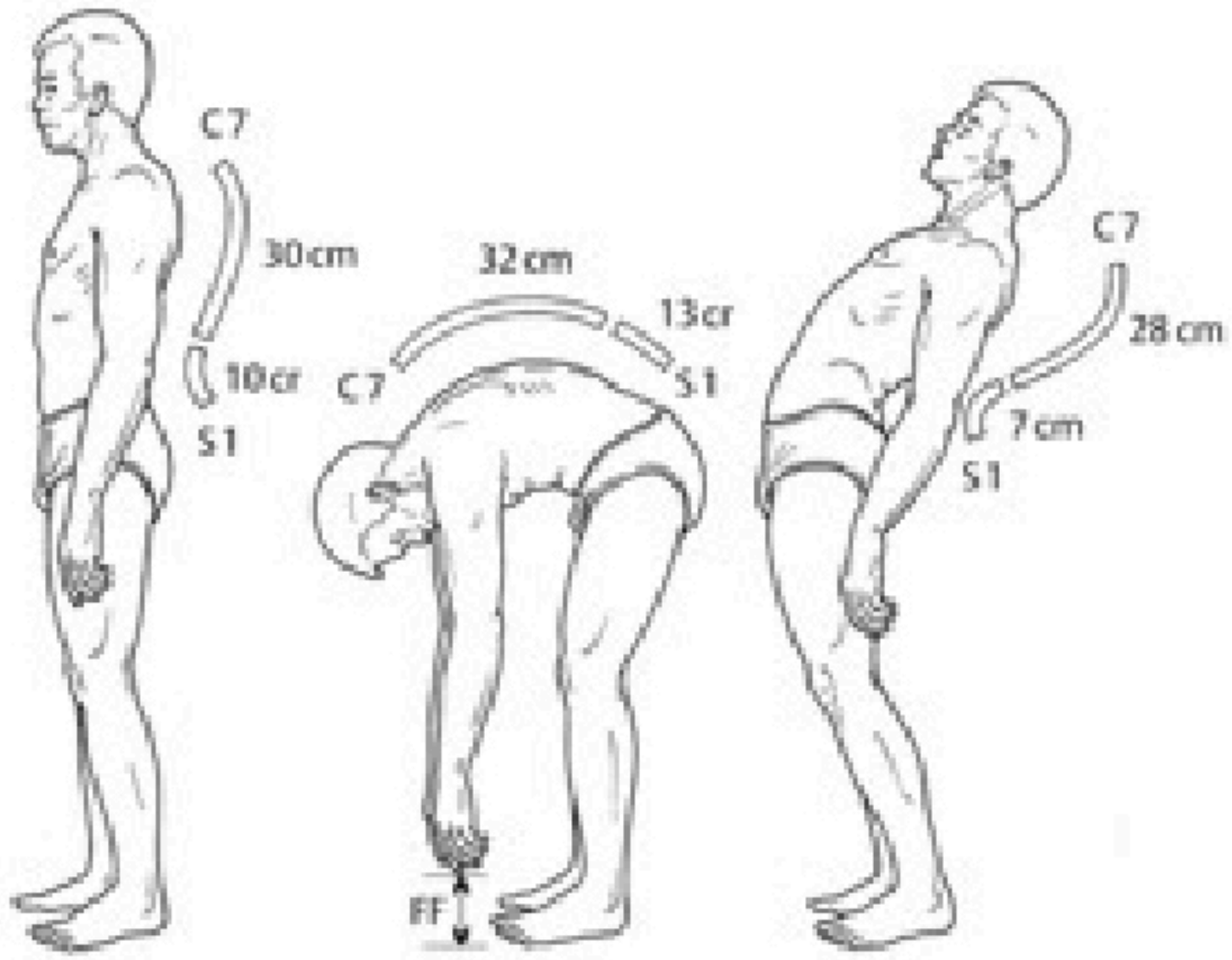

Abb. 1: Schober- und Ott-Zeichen zur Überprüfung der LWS- und BWS-Flexionsfähigkeit (nach [14])

\section{Nervendehnungszeichen}

Hier wird vor allem getestet, ob eine Neurokompression durch vor allem Diskushernien, aber auch durch Frakturen, Tumore/Metastasen oder Abszesse stattfindet. Klassischerweise gehört hierzu die Testung des Lasègue-Zeichens. Hier werden die Nervenwurzeln der Segmente L4-S2 sowie der N. ischiadicus getestet. Das gestreckte Bein des auf dem Rücken liegenden Patienten wird im Hüftgelenk passiv gebeugt. Kommt es bei einem Winkel $<45^{\circ} \mathrm{zu}$ abstrahlenden radikulären Schmerzen des flektierten Beines, spricht man von einem positiven Lasègue-Zeichen (gute Reliabilität [12]), bei Schmerzangabe im kontralateralen Bein von positivem gekreuztem Lasègue. Wird lediglich eine Schmerzangabe lumbal getätigt, ist der Test negativ. Auch Schmerzangabe von $a b>60^{\circ}$ gelten als negativ und sind vermutlich eher durch eine Verkürzung der ischiocruralen Muskulatur hervorgerufen. Wird der Fuss zusätzlich zur Hüftflexion dorsalflektiert, spricht man vom Bragard-Zeichen. Durch die zusätzliche Dorsalflexion wird der Zug auf die Nerven noch verstärkt, die entsprechende Gradzahl wird hier dokumentiert. 
Der umgekehrte Lasègue wird bei dem auf dem Bauch liegenden Patienten durchgeführt, indem das Hüftgelenk passiv überstreckt wird (kann bei Unmöglichkeit der Bauchlage auch in Seitenlage durchgeführt werden). Er testet von L2-L4 und gilt als Dehnungszeichen des N. femoralis, kann aber differentialdiagnostisch auch z.B. bei einer Meralgia paraesthetica positiv sein.

Weitere Nervendehnungszeichen sind das Kernig-Zeichen, bei dem das Knie passiv gestreckt wird bei zuvor flektiertem Hüftgelenk oder auch das Brudzinski-Zeichen, bei dem bei passiver Inklination der HWS reflektorisch die Knie flektiert werden, was der Entlastung der Nervenfasern/Meningen dienen soll. Positiv sind diese Tests bei vor allem meningealer Reizung (Meningismus).

\section{ISG Prüfung - Vorlaufphänomen und Spine-Test}

Rund um das SI-Gelenk gibt es mehrere, teils recht unspezifische Tests. Vorerst sollte es auf Druckschmerzhaftigkeit geprüft werden. Weiterhin gibt es das Mennell-Zeichen, indem bei dem auf dem Bauch liegenden Patienten das SI-Gelenk mit der flachen Hand fixiert wird und dann das entsprechende Bein passiv dorsalextendiert. Bei Schmerzangabe ist der Test positiv. Das 4er-Zeichen (der laterale Malleolus wird auf das kontralaterale Knie gelegt) gilt als positiv bei Schmerzen im ISG oder auch sehr grossem Abstand zwischen Knie und Unterlage (DD Verkürzung Hüftabduktoren). Zudem kann mittels Vorlaufphänomen die Beweglichkeit bzw. das Gleitverhalten der SI-Gelenke getestet werden. Hierzu legt der Untersucher jeweils einen Daumen auf die Spina iliaca posterior superior. Beim Vornüberbeugen wird die Position der Untersucherhände auf Seitengleichheit bzw. auf einseitigen Vorlauf überprüft. Beim Spine-Test wird vom Untersucher dieselbe Handposition eingenommen, der Patient macht jedoch eine Hüft- und Knieflexion. Dieser Test gilt als pathologisch, wenn die Daumenposition des Untersuchers auf gleicher Höhe bleibt (physiologischerweise soll es hier zu einer Absenkung ipsilateral kommen). Schmerzen im Bereich des SI-Gelenkes sind häufig sehr unspezifisch und oft eher als Indikator für eine kranial davon liegende (meist im Bereich der unteren LWS) Pathologie zu sehen, wenngleich SI-GelenksArthrosen existieren sowie auch einige Krankheitsbilder, z.B. der M. Bechterew mit einer Sakroiliitis, hier ihren Ursprung nehmen können.

\section{Neurostatus}

Im Rahmen der Wirbelsäulenuntersuchung ist eine orientierende neurologische Untersuchung zwingend, insbesondere wenn der Verdacht auf eine Neurokompression besteht.

Zuerst gilt es, die Sensibilität aller Dermatome oder auch nur problemorientiert zu untersuchen. Hier ist vor allem der Vergleich zur gesunden Gegenseite zu beachten. Auf etwaige Narben (gestörte Sensibilität durch zerstörte Hautnerven) oder vorbestehende Sensibilitätsdefizite (z.B. bei vorangegangene Diskushernien) muss inspektorisch und anamnestisch geachtet werden. Auch muss sich das Defizit einem/oder mehrerer Dermatome zuordnen lassen. Im selben Zuge sollten auch Kribbelparästhesien oder sonstige Dysästhesien erfragt werden. (Abbildung 2) Im Weiteren erfolgt dann die Kraftprüfung mit Testung aller Kennmuskeln in Kraftgraden nach Janda bzw. dem British Medical Research in M0-5/5. Wichtig hierbei ist, wie ausgeprägt die Parese ist (Kraftgrad), seit wann die Parese besteht (Stunden oder Tage oder länger) und ob sie funktionell relevant ist (Grosszehenheberparese vs. Hüftbeugerparese). Diese Überlegungen bestimmen ganz klar das Therapieregime und dessen Outcome. 
Weiterhin werden die Muskeleigenreflexe getestet. Es ist hierbei auf Auslösbarkeit, Seitenvergleich, evtl. verbreiterte Reflexzonen und pathologische Reflexe zu achten.

Als bekanntester pathologischer Reflex und sog. Pyramidenbahnzeichen sei hier der Babinski-Reflex erwähnt. Durch Bestreichen des lateralen Fussrandes kommt es unter pathologischen Bedingungen zu einer Dorsalextension der Grosszehe bei gleichzeitiger Plantarflexion der übrigen Zehen. Bei Babys ist dieser Reflex im ersten Lebensjahr physiologisch, da die Pyramidenbahnen noch nicht ausgereift sind, bei Erwachsenen jedoch immer pathologisch und bedarf weiterer Abklärung.

Eine spezielle Situation besteht bei einer Conus-Cauda-Symptomatik. Diese kann durch Diskushernien, aber auch Frakturen oder Metastasen hervorgerufen werden. Sie ist immer eine Notfallsituation, da hier wichtige Funktionen wie z.B. die Blasen-/Mastdarmaktivität und die Sexualfunktion nachhaltig und irreversibel geschädigt werden könnten. Hier sind entweder die kaudalen Fasern des Rückenmarks oder die Cauda equina geschädigt. Symptomatisch werden evtl. eine Reithosenanästhesie, Blasen- und Mastdarmentleerungsstörungen, Erektionsstörungen oder ein abgeschwächter Analreflex. Die sofortige weiterführende Diagnostik mittels MRI und bei Bestätigung die operative Entlastung sind in dieser Notfallsituation zwingend so schnell wie möglich durchzuführen. Eine Verzögerung in der Diagnostik gilt quasi als unentschuldbar und muss im Fall des Eintretens zwingend detailliert und nachvollziehbar dokumentiert werden - hohe Entschädigungssummen könnten in Haftpflichtfällen auf den Verursacher einer verzögert durchgeführten Behandlung zukommen. Die Behandlungs- und Folgekosten bei jungen Menschen sind enorm. (Tabelle 4) 


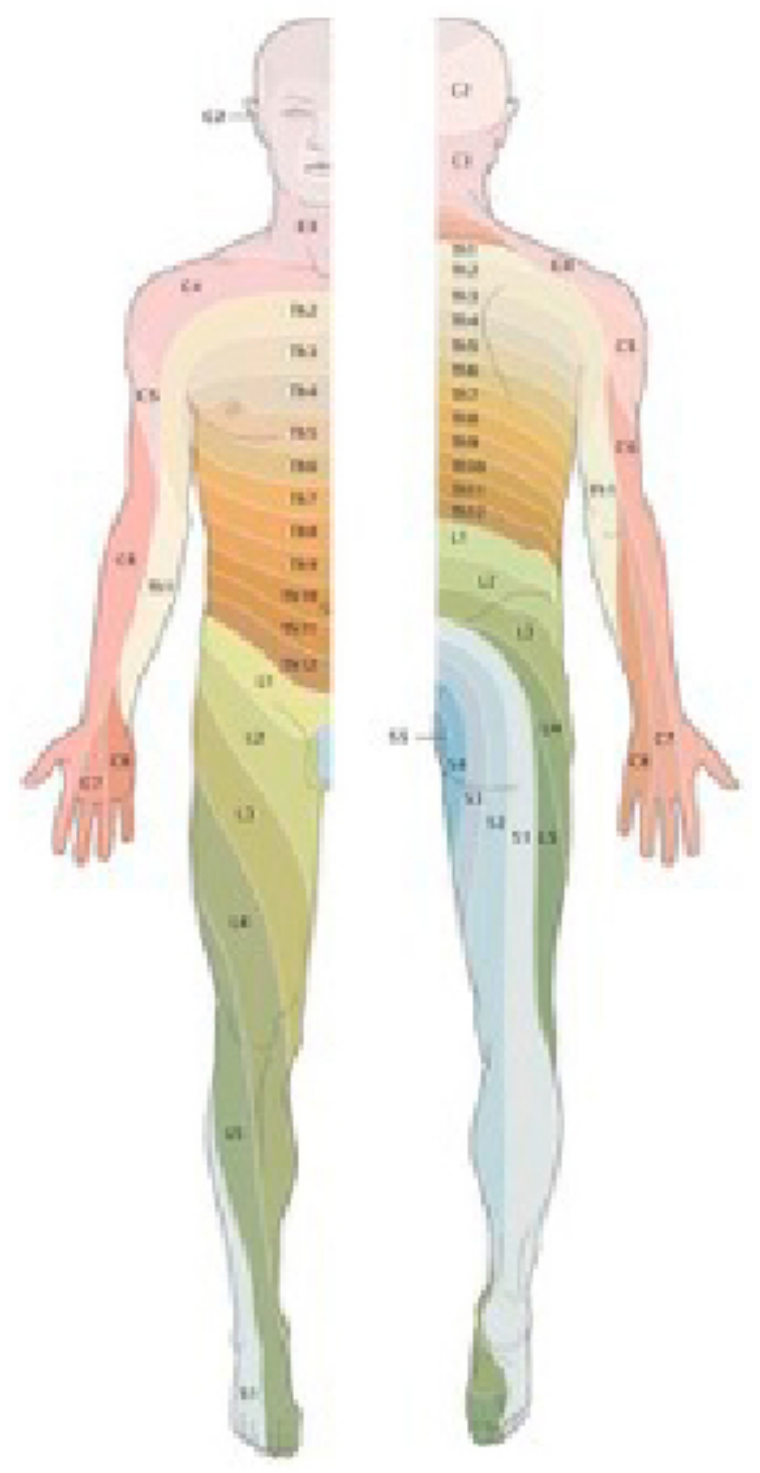

Abb. 2: Dermatomeverteilung [13]

\section{Das Wichtigste in Kürze}

In der Praxis gilt es, die Wirbelsäule zügig in einer strukturierten Untersuchung zu beurteilen, sog. «red flags» und Differentialdiagnosen auszuschliessen sowie die weiterführende Diagnostik schnell, zielgerichtet und effizient einzuleiten. Nur so kann dem Patienten eine suffiziente Therapie angeboten werden. 


\begin{tabular}{|l|l|l|}
\hline Niveau & Kennmuskel & Reflex \\
\hline C5 & M. deltoideus & \\
\hline C6 & M. biceps brachii & BSR \\
\hline C7 & M. triceps brachii & TSR \\
\hline C8 & M. interossei & Horner-Syndrom \\
\hline L2 & Adduktoren, M. iliopsoas & Adduktorenreflex \\
\hline L3 & M. quadriceps femoris & PSR \\
\hline L4 & M. tibialis ant. & PSR \\
\hline L5 & M. EHL, M. glutei & Tibialis post. Reflex \\
\hline S1 & M. triceps surae & ASR \\
\hline M.= Musculus, C=cervical, L=lumbal, BSR=Bicepssehnenreflex, TSR=Tricepssehnenreflex, PSR=Patellarsehnenreflex, \\
ant.=anterior, EHL=Extensor hallucis longus, post=posterior, ASR=Achillessehnenreflex
\end{tabular}

Tabelle 3: Kennmuskeln und Reflexe

\begin{tabular}{|c|c|c|}
\hline Symptom & Verdachtsdiagnose & Abklärung \\
\hline $\begin{array}{l}\text { Lumbalgie mit Anlauf- und Ruheschmerz, } \\
\text { schmerzhaft Reklination }\end{array}$ & Spondylarthrose & Rx, MRI \\
\hline Lumbalgie mit Fieber oder B-Symptomatik & Spondylodiscitis, Tumor & MRI mit KM, CT, Rx \\
\hline Lumbalgie nach Sturz & Fraktur & Rx, CT, evtl. MRI \\
\hline Lumbalgie bei bek. Osteoporose & Osteoporotische Fraktur & Rx, MRI, evtl. CT \\
\hline Radikulopatie & Neurokompression durch z.B. Diskushernie & MRI \\
\hline Kribbel-/Taubheitsgefüht Extremitäten & Neurokompression durch z.B. Diskushernie & MRI \\
\hline Lumboischialgie & $\begin{array}{l}\text { Diskushernie, degenerative Veränderungen, } \\
\text { z.B. Spondylolisthesen }\end{array}$ & MRI, Rx \\
\hline Verminderung der Gehstrecke mit Claudicatio & Spinalkanalstenose & MRI, Rx \\
\hline Nuchalgie & Spondylarthrose & Rx, MRI \\
\hline $\begin{array}{l}\text { Nuchalgie mit Abstrahlung nach occipital, } \\
\text { Schmerzen bei Rotation }\end{array}$ & C1/2-Arthrose & Rx, CT, MRI \\
\hline Nuchalgie mit Fieber & Crowned dens Syndrome & CT, MRI \\
\hline Cervicobrachialgie & Neurokompression durch z.B. Diskushernie & MRI \\
\hline
\end{tabular}


Dr. med. Julia Vavken

Kantonsspital Baden

Wirbelsäulenchirurgie

Im Ergel 1

5404 Baden

julia.vavken@ksb.ch

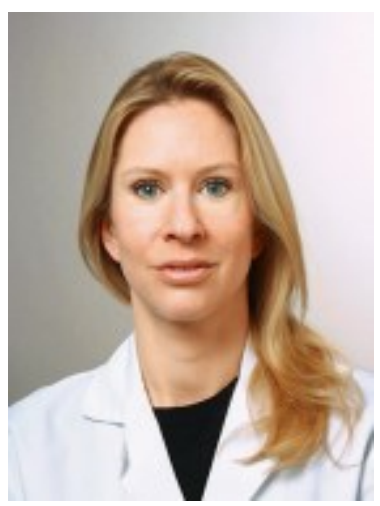

\section{References}

1. Duggleby T, Kumar S. Epidemiology of juvenile low back pain: a review. Disabil Rehabil. 1997 Dec;19(12):505-12.

2. Harvey J, Tanner S. Low back pain in young athletes. A practical approach. Sports Med. 1991 Dec;12(6):394-406.

3. Taimela S, Kujala UM,Salminen JJ, Viljanen The prevalence of low back pain among children and adolescents. A nationwide, cohort-based questionnaire survey in Finland. TSpine Phila Pa 1976). 1997 May 15;22(10):1132-6

4. Watson KD, Papageorgiou AC, Jones GT, Taylor S, Symmons DP, Silman AJ, Macfarlane GJ. Low back pain in schoolchildren: the role of mechanical and psychosocial factors. Arch Dis Child. 2003 Jan; 88(1):12-7.

5. Borg-Stein J, Elson L, Brand E. The aging spine in sports, Clin Sports Med, 2012, Jul;31(3):473-86.doi:10.1016/j.csm.2012.03.002. Epub 2012 Apr 14.

6. Libson E, Bloom RA, Dinari G. Symptomatic and asymptomatic spondylolysis and spondylolisthesis in young adults., Int Orthop, 1982;6(4):259-61.

7. Rossi F. Spondylolysis, spondylolisthesis and sports, J Sports Med Phys Fitness. 1978 Dec;18(4):317-40.

8. Sward L, Hellström M, Jacobsson B., Karlsson L. Vertebral ring apophysis in athletes - Is the etiology different in the thoracic and lumbar spine? Am J Sports Med, 21 (1993) 841-845.

9. Wojtys EM, Ashton-Miller JA, Huston LJ, Moga PJ. The association between athletic training time and the sagittal curvature of the immature spine. AmJSportsMed 4 (2000) 490-498.

10. Measurements in Spine Care, Jens Chapman et al., Thieme New York, 2012.

11. Carson J, Gumpert J, Jefferson A. Diagnosis and treatment of thoracic disc protrusions,J Neurol Neurosurg Psychiatry. 1971;34:68-77.

12. Vroomen PC, de Krom MC, Wilmink JT, Kester AD, Knottnerus JA. Diagnostic value of history and physical examination in patients suspected of lumbosacral nerve root compression. J Neurol Neurosurg Psychiatry. 2002;72:630-634.

13. Prometheus LernAtlas der Anatomie: Allgemeine Anatomie und Bewegungssystem, Sensible Innervation: Dermatome und Hautnervenareale, Michael Schünke, Markus Voll, Karl H. Wesker, Erik Schulte, Udo Schumacher, Thieme 4. Auflage, 2014.

14. Orthopädie und Unfallchirurgie essentials, 2. vollständig überarbeitete und erweiterte Auflage. Stuttgart: Thieme; 2012. Buchholtz S, Wirtz D, Thieme 2. Auflage, 2012. 
